\title{
High-cycle fatigue characteristics of squeezed cast aluminum alloy smooth specimens cut from car wheels
}

\author{
M. Goto ${ }^{1}$, N. Teshima ${ }^{2}$, S. Z. $\operatorname{Han}^{3}$, K. Euh ${ }^{3}$ \& T. Yakushiji ${ }^{2}$ \\ ${ }^{1}$ Department of Mechanical Engineering, Oita University, Oita, Japan \\ ${ }^{2}$ Department of Mechanical Engineering, \\ Oita National College of Technology, Oita, Japan \\ ${ }^{3}$ Korea Institute of Materials Science, Changwon, Republic of Korea
}

\begin{abstract}
High-cycle fatigue tests of smooth specimens cut from squeeze cast $\mathrm{Al}$ alloy car wheels were carried out to clarify the fatigue behaviour under constant stress amplitudes. In addition to this, fatigue tests under two-step stressing were performed to study the effect of stress change on fatigue damage. Fatigue cracks were initiated from eutectic Si particles and sometimes from a slip band in the matrix. But, no cracks were initiated from microscopic defects like pin-holes and shrinkage porosities. The $\ln l$ vs. $N / N_{f}$ relation ( $l$ : crack length, $N_{f}$ : number of cycles to failure) under constant stress amplitudes exhibited stress dependency, namely the crack length initiated at a given value of $N / N_{f}$ was larger in higher stresses. With regard to the fatigue damage under two-step stressing, the cumulative cycle ratio, $\Sigma\left(N / N_{f}\right)$, for low-to-high block stressing was smaller than 1 , however it was larger than 1 for high-to-low block stressing. The value of $\Sigma\left(N / N_{f}\right)$ ) was closely related to the growth characteristics of a small crack.

Keywords: fatigue, squeeze cast aluminum alloy, car wheel, two-step stressing, crack initiation, small-crack propagation, cumulative cycle ratio.
\end{abstract}

\section{Introduction}

Since aluminum casting alloys have superior characteristics such as light-weight, high productivity, cost performance, recyclability, these alloys have been widely used for machine components. A serious problem for component usage of 
casting alloys is a low fatigue strength and less reliability caused from the casting defects like shrinkage cavities peculiar to the casting alloys [1]. Aluminum diecasting technologies dramatically improved the production of high quality castings. Among these technologies, a squeeze casting can achieve excellent mechanical properties, derived from its improvement to refine the microstructure and suppress micro defects such as pinholes, shrinkage porosities [2-4]. Thus, squeeze cast Al alloys are currently applied to a wide variety of products, which include large automobile parts such as cross members and wheels. Recently, many fatigue data of squeeze cast $\mathrm{Al}$ alloys have been reported. Most of those data were gathered from the materials cast for the specimen use. However, there are few data obtained from the specimens cut from actual machine components.

In the present study, fatigue tests of smooth specimens cut from squeeze cast Al alloy car wheels were conducted to clarify the fatigue behaviour under constant cyclic stress amplitudes. The behaviour of cracks was monitored successively by the plastic replication technique. To minimize the scatter in fatigue data due to the fluctuation of laboratory atmosphere, the testing machine was placed in a small cabin in the laboratory. The temperature and humidity in the cabin were kept under control $\left(t=30{ }^{\circ} \mathrm{C}, h=60 \%\right)$. In addition, fatigue tests under two-step stressing were carried out to study the effect of stress change on the behaviour of small cracks.

\section{Experimental procedures}

The material was aluminum cast alloy (JIS-AS4CH). The chemical composition was $7.02 \mathrm{Si}, 0.08 \mathrm{Fe}, 0.37 \mathrm{Mg}, 0.12 \mathrm{Ti}$ and remainder $\mathrm{Al}$. The material was squeeze cast to large wheels for a truck by a vertical squeeze casting machine under the molten metal temperature of $1050 \mathrm{~K}$ and injection speed between 50 and $80 \mathrm{~mm} / \mathrm{sec}$. After the squeeze cast, wheels were heat treated under T6 conditions (solution and aging treatments). The mechanical properties after the heat treatment were $214 \mathrm{MPa} 0.2 \%$ proof stress, $263 \mathrm{MPa}$ ultimate tensile strength, $337 \mathrm{MPa}$ true breaking stress, and $27.3 \%$ reduction of area. Mean dendrite arm spacing was about $30 \mu \mathrm{m}$.

Bar samples for machining the specimen were gathered from a thick portion of the heat treated wheels (Fig.1). Five millimeter-diameters round bar fatigue specimens were machined from these bar samples. The specimen had a shallow circumferential notch $(20 \mathrm{~mm}$ notch radius and $0.25 \mathrm{~mm}$ notch depth), to localize damaged areas for successive observations of the surface. However, the fatigue strength reduction factor for this geometry was close to 1 , so that the specimen can be considered as a plain specimen. Before testing, all fatigue specimens were electrolytically polished $(\sim 20 \mu \mathrm{m}$ from the surface layer) prior to mechanical testing in order to remove any preparation affected surface layer. All tests were carried out at controlled atmosphere $\left(t=30{ }^{\circ} \mathrm{C}, h=60 \%\right)$ using a rotating bending fatigue machine operating at $50 \mathrm{~Hz}$. Observations of fatigue damage on the specimen surface were performed using optical microscopy and scanning electron microscopy (SEM). The crack length, $l$, was measured along the 
circumferential direction of the surface. The measurements of crack length were conducted using a plastic replication technique. The stress value referred to is that of the nominal stress amplitude, $\sigma_{a}$, at the minimum cross section.

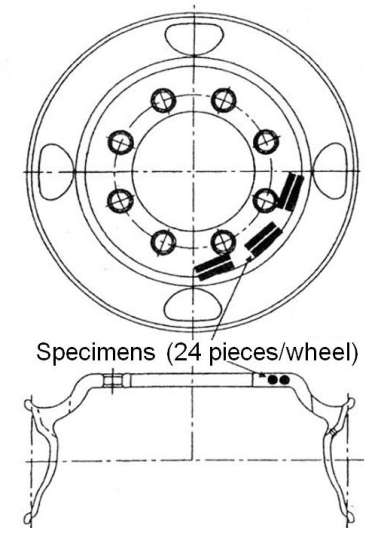

Figure 1: Wheel shape and the location for gathering specimens.

\section{Experimental results and discussion}

\subsection{S-N curve}

Fig. 2 shows the S-N curve in uncontrolled air. Open and solid symbols show the experimental data gathered in summer- and winter-season, respectively. The influence of atmosphere on fatigue strength is clearly recognized. Fatigue life, $N_{f}$, in winter season is about 5 times larger than in summer season. Fig. 3 shows the S-N curve in controlled air. Scatter in the S-N plots for controlled air is drastically reduced when compared to that for the uncontrolled air. The fatigue limiting stress at $10^{7}$ cycles, $\sigma_{w}$, was $110 \mathrm{MPa}$. Consequently, S-N curve for controlled air was comparatively close to that for summer season (dashed line in Fig. 2). To minimize the effect of the fluctuation of laboratory atmosphere on the fatigue strength, all tests were conducted in the controlled laboratory air. Fig. 4 shows the S-N curves and $0.03 \mathrm{~mm}$ crack initiation curves normalized by the ultimate tensile strength, $\sigma_{U}$. The relation for an age hardened Al alloy 6061-T6 (Al-Si-Mg alloy, $\sigma_{U}=309 \mathrm{MPa}$ ) is shown for comparison. The difference in the relations for both materials is negligibly small, whereas the crack initiation life of $\mathrm{AC} 4 \mathrm{CH}$ is slightly longer than that of 6061-T6. Thus, it can be concluded that the fatigue strength of squeeze cast $\mathrm{Al}$ alloy cut from car wheel is comparable to 6061-T6 alloy. This may be because that the specimens were cut from a thick portion in the wheels where includes few defects. Accordingly, the S-N curve does not represent the fatigue strength of actual car wheel itself, however, it is beneficial to the estimation of an average fatigue strength of the material used for car wheels. 


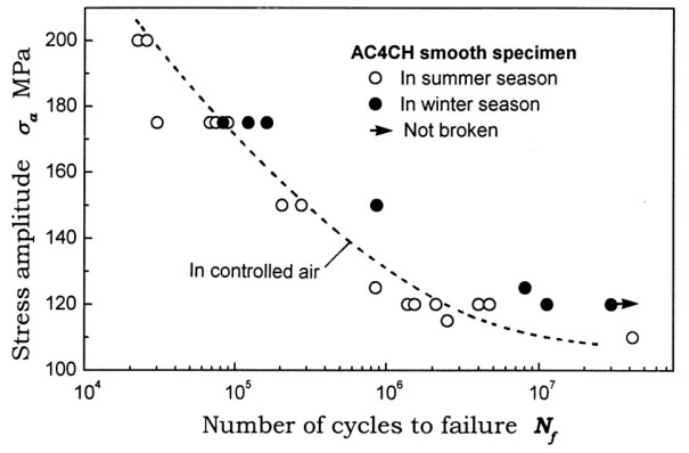

Figure 2: $\quad$ S-N curve in uncontrolled laboratory air.

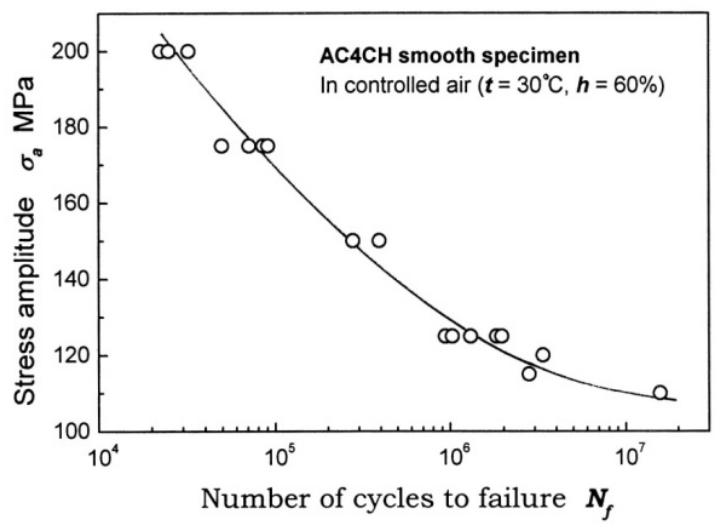

Figure 3: $\quad$ S-N curve in controlled laboratory air.

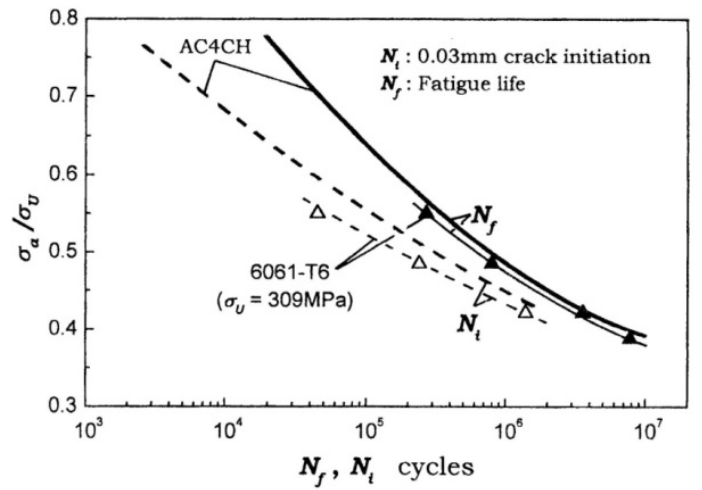

Figure 4: $\quad \sigma_{a} / \sigma_{U}$ vs. $N_{f}$ and $N_{0.03}$ relation for AC4CH and 6061-T6 alloys. 


\subsection{Fatigue behaviour under constant stress amplitudes}

Fig. 5 shows the change in surface states around a major crack which led to the fracture of the specimens. At an early stage of stressing, a crack was initiated at or near the interfaces between the matrix and eutectic Si particles. Fig. 6 shows SEM micrograph of the fracture surface, showing a cluster of eutectic Si particles at the crack initiation site. Another crack initiation sites were slip bands in the matrix, however no cracks initiated from the defects such as pinholes and shrinkage porosities were detected. Fig. 7 shows SEM micrographs of the crack initiation sites: (a) eutectic Si particles and (b) slip bands. After the initiation, the crack growth behaviour was influenced by the microstructure, showing growth paths along eutectic Si particles and slip bands $\left(N=2.25 \times 10^{5}\right.$ in Fig. 5$)$.

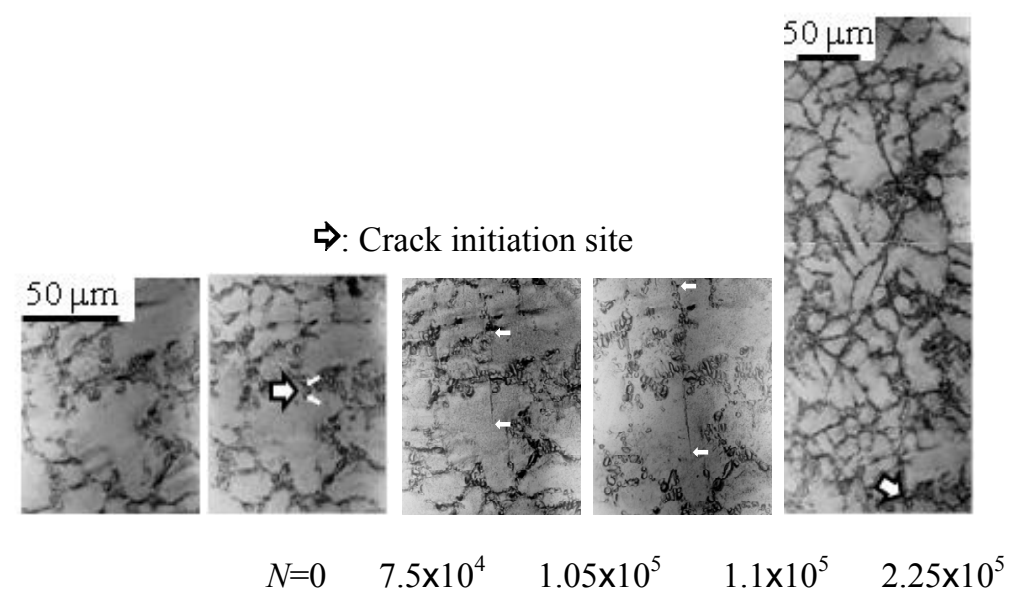

Figure 5: Change in surface state around a major crack.

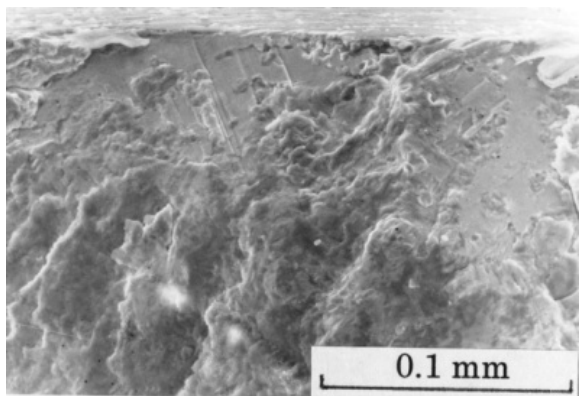

Figure 6: $\quad$ Fracture surface. $\left(\sigma_{a}=125 \mathrm{MPa}\right)$. 


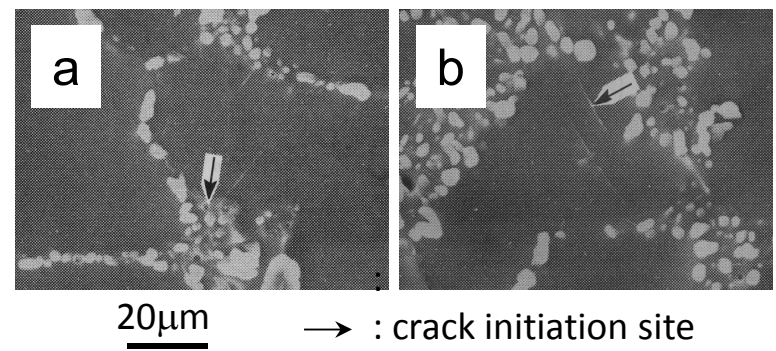

Figure 7: SEM micrographs of crack initiation site; (a) eutectic Si particles, (b) slip bands.

Fig. 8 shows the growth curve $\left(\ln l\right.$ vs. $\left.N / N_{f}\right)$ of major cracks at $\sigma_{a}=175 \mathrm{MPa}$. Although a large scatter in the relation is recognized for the crack length less than $0.3 \mathrm{~mm}$, the relation can be approximated by a curved line. This length 0.3 $\mathrm{mm}$ is nearly equivalent to 10 times of mean dendrite arm spacing. It has been shown that the effect of microstructure on the crack propagation in a squeeze cast $\mathrm{Al}$ alloy can be negligible for the crack length in excess of 8 times dendrite arm spacing [5]. Fig. 9 shows the comparison of the growth curves under different stress amplitudes. The relation depends on the stress amplitude. For a given $N / N_{f}$ value, the higher the stress amplitude, the longer the crack length.

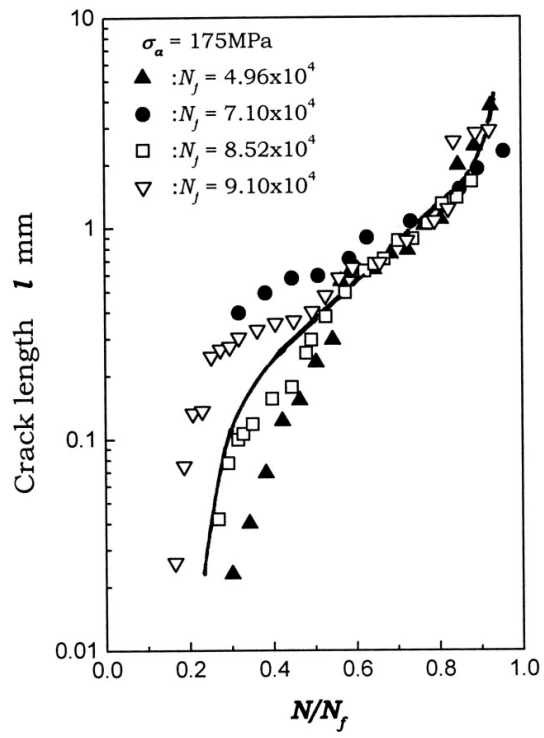

Figure 8: Growth curve $\left(\ln l\right.$ vs. $\left.N / N_{f}\right)$ of major cracks at $\sigma_{a}=175 \mathrm{MPa}$. 


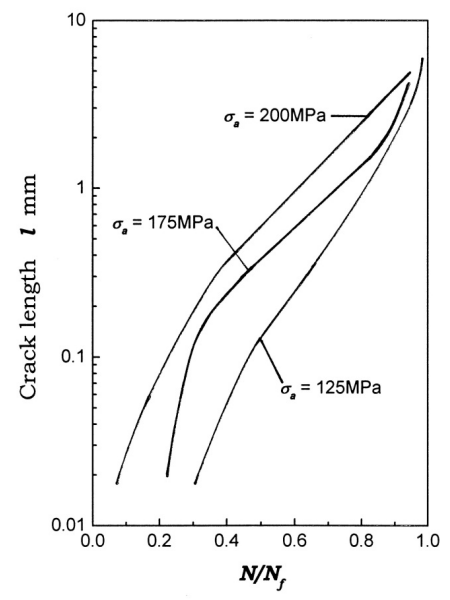

Figure 9: Comparison of the growth curve under different stress amplitudes.

Fig. 10 shows the crack growth curves $\left(\ln l\right.$ vs. $\left.N_{p}\right)$ of three specimens fatigued at $\sigma_{a}=175 \mathrm{MPa}$. Here, the term $N_{p}$ is the number of cycles for cracks between $0.05 \mathrm{~mm}$ in length to fracture length. For the crack length less than $0.3 \mathrm{~mm}$, the growth curves show different slopes by each specimen. For the crack length larger than $0.3 \mathrm{~mm}$, although the growth curves have an abrupt increase in crack length due to crack coalescences, the slope of growth curves excepting the crack coalescence are nearly equivalent each other, showing that the microstructure has negligible effect on the growth behaviour of large cracks.

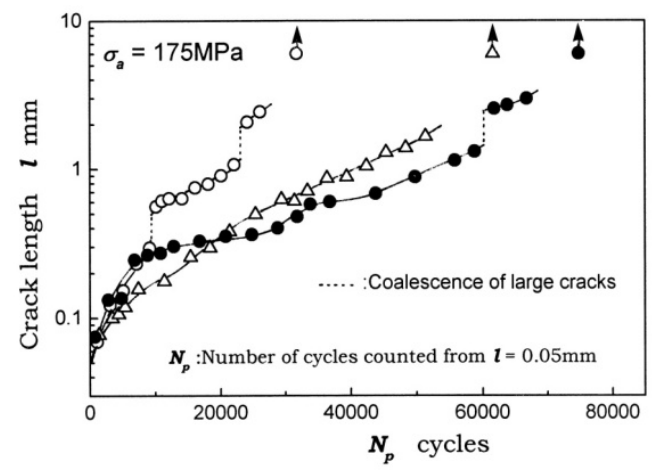

Figure 10: Growth behaviour $\left(\ln l\right.$ vs. $\left.N_{p}\right)$ of three major cracks propagating at $\sigma_{a}=175 \mathrm{MPa}$.

Fig. 11 shows $d l / d N$ vs. $\ln l$ relation. Here, the term $d l / d N$ indicates the growth rate of major cracks calculated from the growth curves represented by smooth curves that pass through the mean average of each set of plots rather than from 
the raw growth data. For all stress amplitudes, the change in the relation occurs at around $l=0.3 \mathrm{~mm}$. The relation for $l>0.3 \mathrm{~mm}$ can be approximated by the straight line. Its slope is about 1 when $\sigma_{a} \gtrsim 150 \mathrm{MPa}$. Meanwhile, the relation for $l<0.3 \mathrm{~mm}$ is accelerated from that expected form an extension of the relation for large crack length $(l>0.3 \mathrm{~mm})$. This acceleration comes from the difference in the crack growth mode, i.e., dominant growth mode tends to change from shear to tensile at $l \cong 0.3 \mathrm{~mm}$. A crack growing with the shear mode is apt to be affected by microstructural inhomogeneity like a slip orientation and grain/phase boundaries, giving rise to a large fluctuation in crack growth behaviour (Fig. 8).

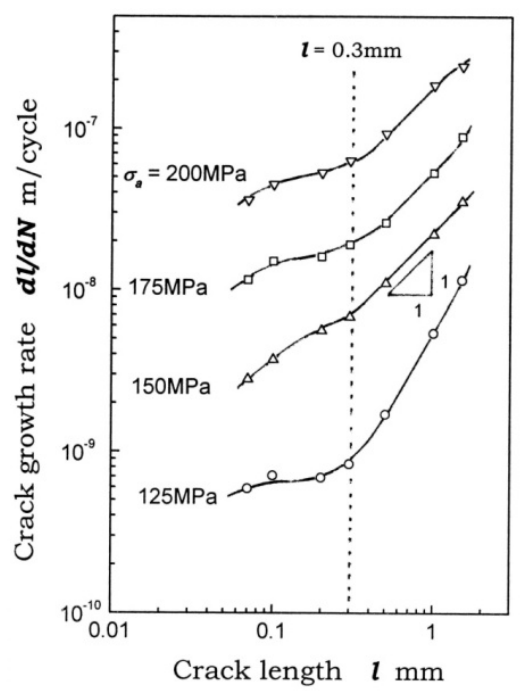

Figure 11: $\quad d l / d N$ vs. $\ln l$ relation.

Fatigue life is mainly controlled by the fatigue crack growth life. Therefore, a quantitative estimation of the service fatigue life of machine components and structures is usually undertaken by applying crack growth laws to members. The propagation for a long (millimeter-range) crack for intermediate $\Delta K$ magnitudes ( $\triangle K$ : stress intensity factor range) is controlled by the Paris law:

$$
\frac{d l}{d N}=C \Delta K^{m}
$$

where $C$ and $m$ are material constants. On the other hand, it is generally accepted that the growth rate of a small crack is more accelerated than that of a long crack with the same $\Delta K$ value [6,7]. This accelerated growth rate results from a difference in the crack closure behaviour between small and long cracks [6].

Nisitani and Goto [9] proposed eqn. (2) for determining the growth rate of small cracks at high stress amplitudes:

$$
\frac{d l}{d N}=D \sigma_{a}^{n} l
$$


where $D$ and $n$ are material constants. The growth data of small cracks in various metals indicated that $d l / d N$ of a mechanically small crack is uniquely determined by the term $\sigma_{a}{ }^{n} l$ and not by $\Delta K[6,9,10]$. Fig. 12 shows the $d l / d N$ vs. $\sigma_{a}{ }^{n} l$ relation $(n=6.6)$. The growth rate of a crack larger than $0.3 \mathrm{~mm}$ propagating under a large stress $\left(\sigma_{\mathrm{a}} \gtrsim 150 \mathrm{MPa}\right)$ is determined by eqn. (2).

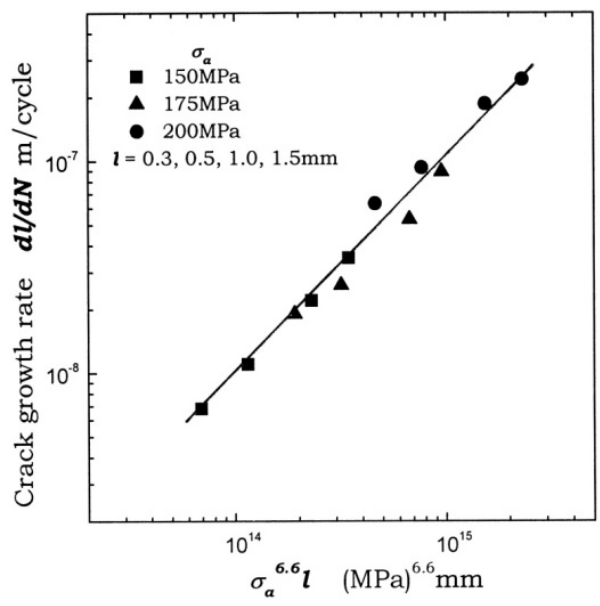

Figure 12: $\quad d l / d N$ vs. $\sigma_{a}^{n} l$ relation $(n=6.6)$.

\subsection{Fatigue damage under two-step stressing}

To clarify the physical background of the effect of stress change on the fatigue damage, two-step fatigue stress tests were conducted, i.e., after an arbitrary number of repetitions at the first stress amplitude, the specimens continued to fatigue at the second stress amplitude. The stresses used for the tests were $\sigma_{a}=$ 125 and $175 \mathrm{MPa}$. The mean fatigue life under constant stress amplitude was $N_{f}$ $=1.409 \times 10^{6}$ cycles (average of 5 specimens) at $\sigma_{a}=125 \mathrm{MPa}$ and $N_{f}=7.42 \times 10^{4}$ cycles (average of 4 specimens) at $\sigma_{a}=175 \mathrm{MPa}$. Fig. 13 shows the crack growth curve for (a) low-to-high and (b) high-to-low block stressing. Arrows indicate the position where the stress was changed from $\sigma_{1}$ to $\sigma_{2}, \sigma_{1}$ and $\sigma_{2}$ being the first and second stress levels, respectively. The dashed lines show the growth curve under constant stress of $\sigma_{2}$ (no repetitions of $\sigma_{1}$ ). For the case of low-tohigh block stressing, there is no significant difference between the growth data after the stress change and the growth curves under the constant stressing. Thus, the crack growth behaviour under $\sigma_{2}$ is not affected by the history of the first stress cycling. On the other hand, for the case of high-to-low block stressing, the growth rate under the second stress is accelerated when compared to that under constant stressing of $\sigma_{2}$. However, when the stress is changed before crack initiation $\left(N_{1} / N_{f 1}=0.204\right)$, no effect of the first stress on the growth behaviour under $\sigma_{2}$ is observed. Although the mechanism for the acceleration of crack growth is not clear, the acceleration may result from an enhanced effective stress 


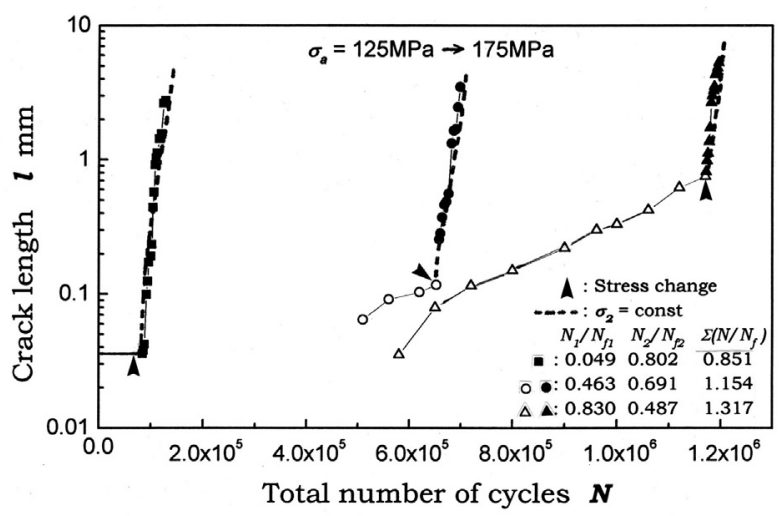

(a) low-to-high block stressing.

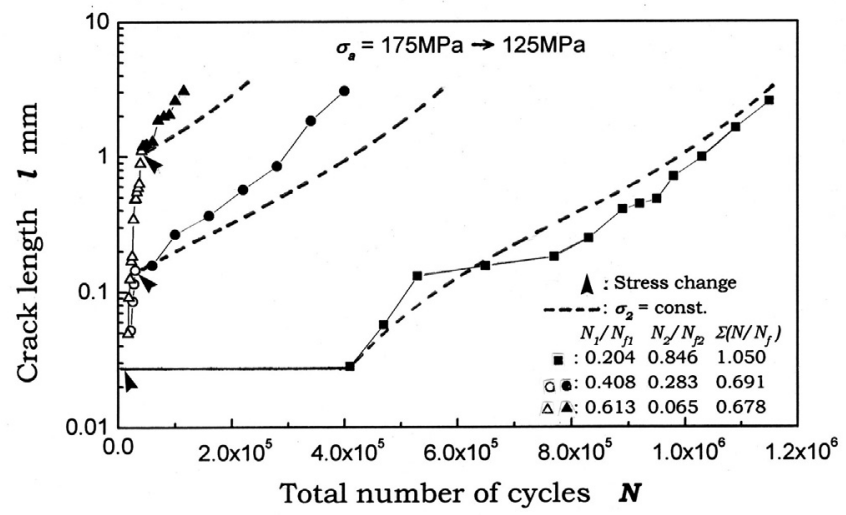

(b) high-to-low block stressing.

Figure 13: Crack growth curve for two-step stressing.

range ratio at a crack tip and cyclic softening at the heavily deformed region around a crack tip caused by the stressing under $\sigma_{1}$.

Fig. 14 shows the value of cumulative cycle ratio, $\Sigma\left(N / N_{f}\right)$; (a) low-to-high and (b) high-to low block stressing. For low-to-high block stressing, values of $\Sigma\left(N / N_{f}\right)$ are larger than 1 . The $\Sigma\left(N / N_{f}\right)>1$ can be explained from no effect of the history of the first stress cycling on the subsequent growth behaviour under $\sigma_{2}$ and the stress dependency of the $\ln l$ vs. $N / N_{f}$ relation (Fig. 9). For high-to-low block stressing, however, the value of $\Sigma\left(N / N_{f}\right)$ is smaller than 1 . The $\Sigma\left(N / N_{f}\right)<1$ results from the acceleration of crack growth after the stress change and the stress dependency shown in Fig. 9. All $\Sigma\left(N / N_{f}\right)$ values for two-step stress fatigue tests fell in the range $0.578<\Sigma\left(N / N_{f}\right)<1.749$. This does not necessary deny the validity of Miner's rule, but there are distinct tendencies of $\Sigma\left(N / N_{f}\right)$ values result from difference in stressing pattern. Thus, when examining the fatigue damage under complex stressing, it should be taken into account that $\Sigma\left(N / N_{f}\right)$ has a 


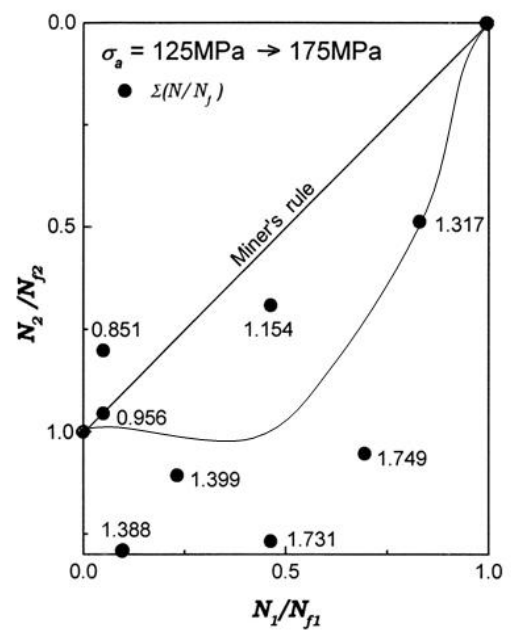

(a) low-to-high block stressing.

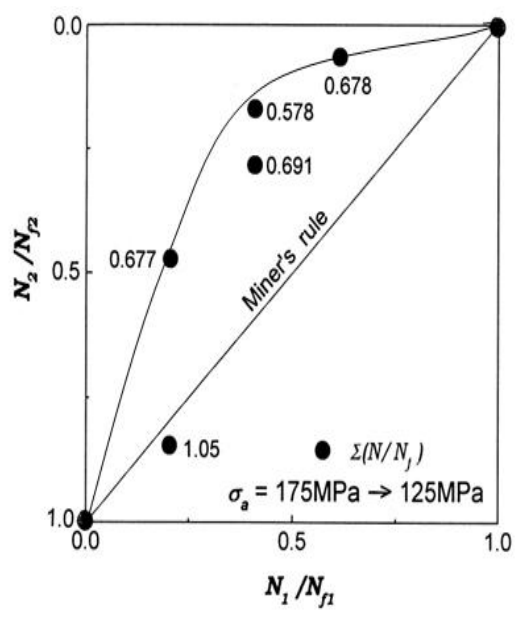

(b) high-to-low block stressing.

Figure 14: Value of cumulative cycle ratio for two-step stressing.

distinct tendency determined by the stressing pattern and stress levels used for the tests.

\section{Conclusions}

Fatigue tests of smooth specimens of AC4CH (Al-Si-Mg alloy) cut from squeeze cast car wheels were carried out to clarify the crack initiation and growth behaviour under constant stress amplitudes and two-step stressing.

The main conclusions may be summarized as follows:

1. The fatigue strength normalized by the tensile strength was comparable to an age-hardened Al alloy 6061-T6 (Al-Si-Mg alloy). This may relate to that the specimens were cut from the thick portion of car wheels where includes few defects.

2. Fatigue cracks were initiated from the interfaces between the matrix and eutectic Si particles, and sometimes from a slip band in the matrix. But, no cracks were initiated from microscopic defects.

3. The $\ln l$ vs. $N / N_{f}$ relation under constant stresses exhibited stress dependency (a crack length at a given $N / N_{f}$ is longer in higher stresses).

4. For a crack less than $0.3 \mathrm{~mm}$, the crack predominantly propagated under the shear mode, showing a microstructure related growth behaviour. After the crack length exceeded $0.3 \mathrm{~mm}$, there was negligible effects of microstructure on crack growth. When the stress was relatively high $\left(\sigma_{a} \gtrsim 150 \mathrm{MPa}\right), d l / d N$ of a crack larger than $0.3 \mathrm{~mm}$ was determined uniquely by a term $\sigma_{a}{ }^{n} l(n=6.6)$.

5. With regard to the fatigue damage under two-step stressing, the cumulative cycle ratio $\Sigma\left(N / N_{f}\right)$ for low-to-high block stressing was smaller than 1 , however it 
was larger than 1 for high-to-low block stressing. This tendency in $\Sigma\left(N / N_{f}\right)$ value was closely related to the growth characteristics of a small crack.

\section{Acknowledgements}

This study was partly supported by a Grant-in-Aid (23560093) for Scientific Research (C) from the Ministry of Education, Science and Culture of Japan as well as the National Research Foundation of Korea (NRF) grant funded by the Korea government (MEST) (No. 2011-0030801)" and by a grant from Integrated Technology of Industrial Materials funded by the Ministry of Knowledge Economy, Republic of Korea. The authors are very grateful to the members of the Strength of Materials Laboratory of Oita University, for their excellent experimental assistant.

\section{References}

[1] Bailey, W.A., Foundry, 93, pp. 96, 1965.

[2] Y. Kaneko, H. Murakami, K. Kuroda and S. Nakazaki, Foundry Trade Journal, 28, pp. 397, 1980.

[3] Williams, G., Foundry Trade Journal, 2, pp. 66, 1984.

[4] Inguanty, P.C., Proc. of the $17^{\text {th }}$ National SAMPE Technical Conf., pp. 61, 1985.

[5] N. Kawagoishi, H. Nisitani, T. Toyohiro, N. Yamamoto and, T. Tsuno, Trans Jpn Soc Mech Eng, A60, pp. 358, 1994.

[6] H. Nisitani, M. Goto and N. Kawagoishi, A small-crack growth law and its related phenomena. Eng Fract Mech, pp. 499, 1992.

[7] M. Goto, H. Nisitani, Fatigue life prediction of heat-treated carbon steels and low alloy steels based on a small crack growth law. Fatigue Fract Eng Mater Struct, 17, pp. 171, 1994.

[8] For example: The behaviour of short fatigue cracks. Edited by Miller KJ, de los Rios ER. EGF-Pub 1, 1986; MEP: Small Fatigue Cracks-Mechanics, Mechanisms and Applications. Edited by Ravichandran KS, Ritchie RO, Murakami Y., Elsevier, 1999.

[9] H. Nisitani, M. Goto, (Eds: Miller, K.J. and de los Rios, E.R.), A smallcrack growth law and its application to the evaluation of fatigue life. The Behaviour of Short fatigue Cracks, Mech Eng Publications LIM: London, pp. 461, 1986.

[10] M. Goto, S. Z. Han, Y. Ando, N. Kawagoishi, N. Teshima and S.S. Kim, WIT Trans Modeling Simulations, 48, pp. 497, 2009. 